\section{Gen relacionado con la sensibilidad a la cocaína}

Gracias a un nuevo estudio llevado a cabo en murinos por un equipo de investigadores de la Universidad de Colombia, se ha ampliado el conocimiento de la función que desempeña la herencia genética en la adicción a diferentes sustancias. El estudio, financiado por el Instituto Nacional del Abuso de Drogas (NIDA) de los Estados Unidos de América y dirigido por el doctor René Hen, ha revelado que los ratones que no tienen el gen de los receptores de la serotonina son más susceptibles a la atracción de la cocaína que los ratones que lo tienen. Habiendo estudios anteriores sugerido que la respuesta a las drogas es afectada - sin saber cómo- por la actividad de la serotonina, los investigadores crearon por alteración genética una estirpe de ratones que carecen de uno de los receptores de la serotonina, el 5-HT(1b). Se observó que los ratones sin el gen eran más sensibles que los demás tanto a los efectos motores como a los de gratificación de la cocaína. Estos se han llegado a conocer como los ratones "noqueados", pues aprendieron a autoadministrarse cocaína mientras los científicos determinaban cuál era su límite de saciedad. El de esos ratones resultó ser mucho más alto que el de sus controles salvajes. Por el contrario, el límite de saciedad con los alimentos fue similar en ambos grupos de ratones. Cuando los ratones fueron llevados al aire libre y se les permitió obtener cantidades cada vez mayores de cocaína, los noqueados respondieron más activamente y exhibieron el comportamiento inducido por la cocaína a dosis más bajas que los salvajes.

Buscando los posibles cambios encefálicos que pudieran explicar la preferencia por la cocaína de los ratones noqueados, el equipo de científicos descubrió concentraciones elevadas de una proteína precursora (FosB) que aumenta cuando hay exposición crónica a la cocaína y se considera crítica en los efectos motores y de gratificación producidos por la droga. Los investigadores piensan que los ratones noqueados pueden responder de forma semejante a otras drogas que causan adicción. Ya se ha observado que gustan de autoadministrarse alcohol y que su comportamiento es más impulsivo que el de otros ratones, rasgo que se asocia a menudo con el abuso de drogas.

"Es cada vez más evidente", declaró el doctor Alan I. Leshner, director del NIDA, "que el perfil ge- nético individual es un factor primordial que determina la vulnerabilidad a la drogadicción. Descubrir cuáles son los genes relacionados con las adicciones nos lleva mucho más cerca de poder establecer nuevas estrategias para prevenir y tratar esos problemas". El NIDA ha lanzado una iniciativa importante para estudiar la vulnerabilidad de origen genético a las drogas. Dada la complejidad del problema, en esa iniciativa se empleará una variedad de estrategias en las que se incluye la cartografía genética de seres humanos y de animales. (National Institute of Drug Abuse. Scientists identify gene involved in sensitivity to cocaine [comunicado de prensa]. 13 de mayo de 1998; Rocha BA, Scearce-Levie K, Lucas JJ, Hiroi N, Castanon N, Crabbe JC, et al. Increased vulnerability to cocaine in mice lacking the serotonin1B receptor. Nature 1998; 393:175-178.)

\section{La desigualdad social y las tasas de mortalidad en Barcelona}

Aun en países con buenos programas de bienestar social se observan desigualdades en el acceso a los servicios de salud. Esas desigualdades aumentan a medida que mejora la salud general de las personas pudientes. En las grandes ciudades hay vecindarios de gente muy rica $\mathrm{y}$ de gente muy pobre y esas diferencias se reflejan en las tasas de mortalidad. Un estudio reciente sobre este tema se llevó a cabo en Barcelona, España, ciudad de 1650000 habitantes donde se ha estudiado la ecología de la desigualdad en la mortalidad, pero no su tendencia. Con objeto de conocer esa tendencia, se compararon las tasas de mortalidad de distritos electorales de bajo nivel socioeconómico con las de otros distritos durante el período de 12 años de 1983 a 1994. De los 38 distritos electorales que comprende la ciudad, 11 (con una población de 300000 personas) se consideraron de nivel socioeconómico bajo y abarcaban las zonas centrales más antiguas de la ciudad además de otros distritos periféricos. Los 27 restantes tenían una población de 1400000 habitantes.

Se obtuvieron datos de los certificados de defunción y direcciones postales del censo municipal, el cual está vinculado con el registro de mortalidad. Las causas básicas de muerte se codificaron según la Clasificación Internacional de Enfermedades (CIE 9). 
Se estudiaron la tuberculosis, el sida, todas las enfermedades infecciosas incluido el sida, tumores malignos del pulmón, todos los tumores, enfermedad isquémica del corazón, enfermedades cerebrovasculares, todas las enfermedades del aparato circulatorio, todas las enfermedades del aparato respiratorio, cirrosis, todas las enfermedades del aparato digestivo, sobredosis de drogas y envenenamientos, y todas las causas externas. Se computaron tasas de mortalidad estandarizadas por edad según el sexo y año en cada distrito electoral, así como las tasas estandarizadas de años de vida potencial perdidos (AVPP). Se tomó como población estándar toda la población barcelonesa por sexo. En los cálculos de los AVPP se incluyeron las defunciones de personas de 1 a 70 años de edad. También se calcularon las tasas específicas de mortalidad por edad. La evolución de la mortalidad en cada grupo de distritos se describió mediante un modelo de regresión lineal.

Durante los 12 años estudiados, las tasas de mortalidad general fueron habitualmente más altas en los distritos de bajo nivel socioeconómico. En ellos, la mortalidad por todas las causas disminuyó de 1056,4 muertes por 100000 habitantes en 1983 a 1012,1 en 1994; en los demás distritos la disminución fue de 838,2 a 809,8. La desigualdad aumentó en relación con algunas causas como el sida, que empezó a manifestarse con un solo caso en 1986 y se incrementó en los distritos pobres $(4,4 \%$ de las defunciones en 1994) en comparación con los restantes (1,9\% de las defunciones). La desigualdad aumentó también con las muertes causadas por sobredosis de drogas. El primer caso se dio en 1984 y 12 años más tarde representó $1,2 \%$ de las defunciones en los distritos pobres y $0,5 \%$ en los demás. A esas dos causas de muerte se puede atribuir el aumento de desigualdad social asociado con muerte prematura, sobre todo en los grupos de edad entre los 15 y 44 años. La tercera causa de aumento de desigualdad fue el cáncer de pulmón en los hombres. Entre los distritos más pobres y los demás se notó una reducción en enfermedades cardiovasculares, pero esa ventaja se anula frente al aumento de la desigualdad relacionada con la mortalidad prematura debido al sida y las sobredosis de drogas. Es de notar que en Barcelona, $52 \%$ de los casos de sida correspondieron a usuarios de drogas por vía intravenosa.

Los resultados de estudios como este implican que las políticas de salud pública no son suficientemente equitativas. Los autores recomiendan que se tome acción en cuatro direcciones: fomentar el conocimiento y la motivación individuales y mejorar las habilidades personales, lo cual suele llevar a un cambio de los comportamientos de riesgo; estimular la formación de redes sociales comunitarias contra riesgos de la salud; mejorar el acceso a insta- laciones y servicios, lo que implica una mejora de las condiciones de vida y trabajo; y procurar cambios macroeconómicos y culturales para reducir la pobreza y la desigualdad en la sociedad. (Borrel C, Plasènsia A, Pasarin I, Ortún V. Widening social inequalities in mortality: the case of Barcelona, a southern European city. I Epidemiol Community Health 1997; 51(6):659-667.)

\section{Los suplementos de cinc mejoran el crecimiento de niños guatemaltecos}

El retraso del crecimiento es un problema que prevalece hasta en $58 \%$ de la población de Guatemala. Si bien se sabe que las infecciones y la nutrición inadecuada tienen mucho que ver con ello, recientemente se han considerado las consecuencias específicas de la carencia de ciertos nutrientes; en particular, del cinc. En las zonas rurales del país, la dieta de los niños se basa principalmente en tortillas de maíz con alto contenido de fitato y calcio, lo que perjudica la absorción del cinc. Además, casi nunca consumen productos de origen animal, principales fuentes de cinc. Para poner a prueba la hipótesis de que la falta de cinc inhibe el crecimiento de esos niños, se diseñó un ensayo doble ciego basado en la comunidad rural de Santa María de Jesús, en la zona central de Guatemala. Se estudiaron los efectos del cinc en el crecimiento y la composición corporal de 89 lactantes guatemaltecos de 6 a 9 meses de edad. Estos se asignaron aleatoriamente a recibir $4 \mathrm{~mL}$ de una bebida que contenía $10 \mathrm{mg}$ de sulfato de cinc $(n=45)$ o placebo $(n=44)$ diariamente por un período de 6,9 meses. Se tomaron medidas antropométricas de base, que luego se repitieron a intervalos de uno o dos meses en el período de estudio. También se obtuvieron datos antropométricos maternos así como de las características socioeconómicas y demográficas de las familias.

La suplementación con cinc se asoció con un aumento general de $0,61 \mathrm{~cm}^{2}$ en el brazo $(P=0,02)$ y un aumento de estatura cuya media fue de $0,75 \mathrm{~cm}$ más que los que no tomaron cinc $(P=0,12)$. Sin embargo, hubo una interacción significativa entre el grupo tratado y la estatura inicial según la edad $(P=$ $0,04)$, de manera que los lactantes que recibieron cinc y eran demasiado pequeños para empezar aumentaron 1,4 cm más que los niños demasiado pequeños que recibieron placebo. Ese incremento tiene importancia biológica porque equivale a un poco más de la mitad de una desviación estándar del crecimiento lineal esperado en niños de esa edad o $15 \%$ del crecimiento en estatura esperado de los 7 a los 14 meses.

Se concluyó que la suplementación con cinc de ese grupo de lactantes rurales guatemaltecos du- 
rante 6,9 meses aumentó la acumulación de masa magra y mejoró el crecimiento lineal de los que estaban demasiado pequeños al comenzar el estudio. Se necesitan estudios ulteriores para determinar si la suplementación con cinc durante períodos más largos puede tener efectos más intensos y generalizados en su crecimiento físico. (Rivera JA, Ruel MT, Santizo MC, Lönnerdal B, Brown KH. Zinc supplementation improves the growth of stunted rural Guatemalan infants. J Nutr 1998;128:556-562.)

\section{¿Existe una relación entre el colesterol y la violencia?}

Hoy día incumbe cada vez más a los médicos de atención primaria tener en cuenta la violencia, que ha llegado a considerarse un problema de salud pública. La violencia no solo es una causa principal de morbilidad sino también de muerte en el grupo de personas menores de 44 años y de años de vida perdidos a todas las edades. Por otra parte, el problema común del exceso de colesterol en la sangre suscita grandes debates relacionados con el costo, los riesgos y los beneficios del tamizaje de grupos de población y su tratamiento. Uno de los riesgos posibles tiene que ver con la relación que parece existir entre la reducción o las concentraciones bajas de colesterol y la muerte violenta en hombres. A pesar de que esta presunta asociación se ha criticado porque no es biológicamente verosímil, en un estudio muy reciente se hace una revisión de la literatura para determinar si esa relación es coherente con los criterios de causalidad de Austin Bradford Hill y si la validez del constructo está respaldada por los resultados de distintos tipos de estudios.

Utilizando como fuentes las bases de datos y bibliografías MEDLINE, PsycINFO y Current Contents, se buscaron artículos publicados en inglés desde 1965 hasta 1995. En la búsqueda se usaron las palabras clave colesterol y violencia y colesterol y suicidio. Se seleccionaron solamente trabajos de observación y experimentales, así como metanálisis sometidos al arbitraje de expertos, que presentaran investigaciones originales; relacionaran las concentraciones de colesterol con violencia definida conductualmente; $y$, de ser experimentales, que tuvieran una sola intervención relacionada con lípidos. Se encontraron 163 artículos que vinculaban el colesterol con la violencia y se seleccionaron 32 que cumplían con los criterios de inclusión en el estudio. Estos se agruparon por tipo de estudio y luego se extrajeron de cada uno los datos sobre la relación entre la violencia y la concentración de colesterol.

En la síntesis de datos, los estudios obervacionales -incluidos los de cohorte, casos y controles y transversales- mostraron repetidamente un aumento de muertes y comportamientos violentos en personas con concentraciones bajas de colesterol. Algunos metanálisis de ensayos aleatorios señalaron un exceso de muertes violentas en hombres sin cardiopatías que se asignaron aleatoriamente a terapias para reducir el colesterol. Otros estudios de tipo experimental mostraron que los comportamientos violentos aumentaban en monos sometidos a dietas de muy bajo contenido de colesterol. La investigación tanto en seres humanos como en animales indica que las concentraciones bajas de colesterol reducen la actividad central del neurotrasmisor serotonina, que a su vez está relacionado causalmente con el control de la conducta violenta. Durante el estudio se hicieron búsquedas adicionales para relacionar la serotonina con el colesterol y con la violencia. $\mathrm{Mu}-$ chos ensayos respaldaron la hipótesis de que hay una relación significativa entre la violencia y las concentraciones bajas o reducción del colesterol $(P=$ $0,001)$. Los datos sobre esta asociación coinciden con los criterios establecidos por Hill para reconocer una asociación causal. Por lo tanto, en los análisis de riesgo-beneficio sobre el tamizaje y tratamiento del colesterol debe tenerse en cuenta el riesgo que plantea la conducta violenta. Los resultados de este estudio apoyan una actitud conservadora en el manejo de las personas hipercolesterolémicas que están en riesgo bajo o moderado de muerte por cardiopatía. Además, es preciso hacer investigaciones sobre la forma en que subfracciones de lipoproteínas se relacionan con la violencia: si son factores demográficos, conductuales o bioquímicos los que influyen en la susceptibilidad a la violencia asociada con el colesterol bajo y guían la evaluación de factores de riesgo (edad, sexo, consumo de alcohol, antecedentes psiquiátricos, medidas neuroquímicas o de personalidad), y si las drogas serotonérgicas atenúan el riesgo de violencia en personas que son candidatas al tratamiento para reducir el colesterol. En el futuro, debe estudiarse la asociación entre la reducción del colesterol y enfermedades de todo tipo y no solo cardiopatías y establecerse estrategias para evaluar cuantitativamente los riesgos y beneficios del tratamiento de la hiperlipidemia, según las características de cada paciente. (Colomb BA. Cholesterol and violence: is there a connection? Ann Intern Med 1998;128:478-487.)

\section{Verosimilitud biológica en la inferencia causal}

Gran parte de la investigación sobre el cáncer se ocupa de vincular diversas exposiciones - agentes infecciosos, factores ambientales, riesgos ocupacionales, estilos de vida, uso de medicamentos y 
tecnologías, etc.-, con sus posibles consecuencias morbosas. Algunas exposiciones, como el hábito de fumar, son bien conocidas; otras siguen siendo tema de controversia, y aun otras han sido refutadas como factores de riesgo. Entre la investigación de esas exposiciones y la aplicación exitosa de intervenciones preventivas se lleva a cabo el proceso de inferencia causal, en el que se evalúa, generalmente en trabajos de revisión y editoriales, la información disponible. La práctica actual de inferencia causal se basa en dos grupos de criterios: los propuestos por el Director General de Sanidad de los Estados Unidos en 1964 y por Austin Bradford Hill en 1965. Los avances biológicos y su integración con la ciencia de la salud pública en la epidemiología molecular han impartido mucha importancia al criterio causal de verosimilitud biológica. Por eso es de especial interés el comentario que aquí se resume y que constituye un trabajo de revisión de la función de la verosimilitud biológica en la inferencia causal tal como se describe en la literatura metodológica y se usa en la práctica. Por supuesto, debe entenderse que la verosimilitud biológica es solo uno entre diversos criterios que es necesario tener en cuenta en la inferencia causal y que esa verosimilitud depende de los conocimientos biológicos del momento. A lo largo de los años se han destacado tres formas de pensar en lo que es biológicamente verosímil: 1) una hipótesis sobre un mecanismo causal razonable, para el cual no hay pruebas biológicas; un mecanismo razonable y pruebas biológicas que respalden su causalidad; y 3) lo anterior, más pruebas de cómo el factor influye en un mecanismo conocido de la enfermedad. Los autores estudiaron la forma en que estos abordajes aparecen en investigaciones de la relación entre el hábito de fumar y el cáncer cervicouterino, y entre la vasectomía y el cáncer de próstata. En los trabajos publicados durante los años ochenta y noventa sobre el tabaquismo y el cáncer cervicouterino, algunos revisores hicieron caso omiso de algunas o de todas las hipótesis biológicas presentadas y usaron definiciones distintas de verosimilitud biológica. Muchos autores llegaron a inferir causalidad sin pruebas biológicas que apoyaran la hipótesis. Sin embargo, por lo menos uno empleó una definición más estricta de la verosimilitud biológica. Ningún autor mencionó ni describió de modo alguno un modelo básico de carcinogénesis que explicara cómo las pruebas biológicas citadas se relacionaban con ese proceso.

Los estudios sobre morbilidad y mortalidad de hombres que habían tenido vasectomías empezaron a aparecer a finales de los años setenta y principios de los ochenta y en ese decenio se publicaron varios informes de investigación. Uno de casos y controles mencionó la posible relación entre la vasectomía y el cáncer de próstata. De 1990 en adelante proliferaron los estudios sobre ese tema y más de 20 trabajos enfocaron los mecanismos biológicos. En general, el tratamiento de las nociones biológicas siguió más o menos el mismo patrón que la literatura sobre el tabaquismo y el cáncer cervicouterino. Los autores examinaron diversos mecanismos biológicos, pero citaron pruebas que variaban considerablemente. Ninguno explicó cómo abordaba el concepto de verosimilitud biológica ni describió las reglas de inferencia para ese criterio. La única e importante diferencia fue que ninguno afirmó que existiera causalidad. La falta de pruebas biológicas para los mecanismos aducidos fue un argumento común contra la afirmación de que la vasectomía causara el cáncer de próstata (o aun fuera un factor de riesgo) a pesar de los resultados de los estudios epidemiológicos.

En ninguno de estos dos campos de estudio tan divulgados se encuentra nada remotamente parecido a un conjunto de reglas coherente que permita hacerse una opinión sobre la verosimilitud de las pruebas biológicas. Es más, tal parece que nunca se hayan propuesto ni en teoría ni en la práctica esas reglas tan necesarias. Evidentemente, es esencial dar un paso adelante en ese sentido y la primera recomendación es mejorar la calidad de las revisiones de literatura y los metanálisis. En estos trabajos deben darse a conocer a los lectores todas las explicaciones y mecanismos biológicos anteriormente propuestos. Por supuesto, los revisores pueden proponer nuevos mecanismos o excluir alguna que otra hipótesis biológica, pero en este caso deben decir claramente en la sección de métodos por qué la excluyeron. Una revisión de calidad debe declarar cómo y con qué criterios y reglas probatorias se ha hecho la evaluación de causalidad y, como se ha dicho, ese es el elemento que falta. Además, formar un juicio sobre alguna exposición y su efecto carcinógeno puede depender en parte de ciertos aspectos de una situación. Por otra parte, si en el caso del cáncer se conocen procesos que son casi universales, es decir, que ocurren con diversos tipos de tumores, es muy posible formular algunas reglas generales. Estas se basarían en los conocimientos cada vez más amplios sobre la biología del cáncer combinados con teorías generales de metodología y razonamiento científico. Según los fundamentos de la ciencia actual, biología se refiere arbitrariamente a sucesos internos de un organismo. Lo que le sucede a un individuo o a una población se llama conductual o social, respectivamente. Sin embargo, en la biología del cáncer humano hay un gran número de gradientes de explicación del mecanismo; piénsese, por ejemplo, en el acto socialmente mediado de fumar, que luego puede desglosarse en sus muchos efectos biológicos. Así que el segundo gran campo que re- 
quiere mejora es el uso de los conocimientos más actualizados sobre los complejos sistemas que interactúan en la biología del cáncer para proponer nuevas reglas aplicables al amplio abanico de resultados de investigación que se examinan al realizar una inferencia causal. (Weed DL, Hursting SD. Biologic plausibility in causal inference: current method and practice. Am J Epidemiol 1998;147:415-425.)

\section{Regulación de la ovulación con feromonas humanas}

Las feromonas son sustancias liberadas en el ambiente por cada individuo y percibidas mediante el olfato por otros individuos de la misma especie, en quienes los componentes químicos provocan un cambio físico o de comportamiento. Hasta hace poco no se había comprobado que existieran feromonas humanas, es decir, compuestos que regulan ciertos mecanismos neuroendocrinos sin ser conscientemente detectados como olores. Los primeros indicios de su existencia se derivaron de haberse ponderado el fenómeno de la sincronización del ciclo menstrual en grupos de mujeres que viven juntas. En las ratas ocurre una sincronía ovárica similar en la que intervienen dos feromonas diferentes. Una, que se produce antes de la ovulación, acorta el ciclo ovárico; la segunda, que se produce durante la ovulación, alarga el ciclo. Estas feromonas fueron predichas por un modelo de oscilador acoplado de sincronía ovárica. Por simulación computadorizada se demostró que las feromonas no solo eran capaces de producir sincronía, sino también otros efectos observables de asincronía y estabilización cíclica. Aplicando el mismo modelo a los seres humanos, puede mostrarse un mecanismo feromonal potencial de sincronía menstrual y otras formas de regulación social de la ovulación.

En un estudio de 29 mujeres de 20 a 35 años de edad, se recogieron compuestos de las axilas de nueve de ellas en diferentes fases hormonales del ciclo menstrual, se mezclaron con alcohol y se aplicaron a las 20 restantes debajo de la nariz. Ninguna de las participantes estaba al tanto de la hipótesis a prueba ni del origen de los compuestos, pues pensaban que el estudio pretendía buscar formas no invasoras de detectar la ovulación. Todas dijeron que los compuestos olían a alcohol y nada más. Las donantes proporcionaron todas las noches muestras de orina, que se examinaban para detectar la hormona luteinizante que desencadena la ovulación y junto con otros datos sobre las secreciones vagina- les, la menstruación, la temperatura basal de cuerpo y el aumento de la glucurónida de progesterona en la fase lútea postovulatoria, permitían distinguir los compuestos axilares de la fase folicular de los de la fase ovulatoria. Los compuestos se aplicaron a diario, por no saberse en qué momento del ciclo menstrual las mujeres son más sensibles a las feromonas. En las ratas, ello ocurre a mitad del ciclo (cuando ovulan), período en el cual las mujeres son especialmente sensibles a ciertos estímulos olfatorios.

Las destinatarias tuvieron ciclos de 1 a 14 días más cortos después de recibir los compuestos axilares producidos en la fase folicular del ciclo menstrual de las donantes y ciclos de 1 a 12 días más largos cuando recibían compuestos ovulatorios, que representan efectos opuestos. Esas respuestas se manifestaron al primer ciclo de exposición aunque se variara la secuencia de presentación de los compuestos. Las reacciones descritas indican que los compuestos contenían feromonas y que se trataba de dos clases distintas como en las ratas. La exposición a los compuestos axilares solo alteró la fase folicular, lo cual sugiere que las feromonas dependen de los ovarios y tienen efectos opuestos en la ovulación porque cambian de forma diferencial el ritmo de maduración folicular o umbral hormonal que desencadena el aumento de hormona luteneizante. Es así como se produce la sincronía, la asincronía o la estabilización del ciclo menstrual en un grupo social.

Es posible que, como en el caso de otras especies, las feromonas humanas se produzcan en las glándulas apocrinas (activas solamente en el período de madurez sexual), en las ecrinas (que producen sudor, el cual contiene compuestos que se encuentran también en la saliva y la orina), en las células epiteliales o por acción bacteriana. Los compuestos se tomaron de las axilas porque tienen las cuatro fuentes potenciales de feromonas y también porque en dos intentos anteriores de estudiar el tema se usaron compuestos axilares. No se sabe si las feromonas de los seres humanos tienen tantas consecuencias como en los animales y pueden afectar, como en las ratas, la edad de la pubertad, los intervalos intergenésicos, la edad de la menopausia y la concentración crónica de estrógeno durante la vida de una mujer. En otras especies hay tipos de feromonas que no dependen de la función ovárica y determinan la preferencia de los hámsters en el apareamiento sexual, las relaciones de dominancia entre los elefantes machos en celo y muchos otros aspectos de la interacción social en mamíferos. (Stern K, McClintock MK. Regulation of ovulation by human pheromones. Nature 1998;392:177-179.) 